This item was submitted to Loughborough's Research Repository by the author.

Items in Figshare are protected by copyright, with all rights reserved, unless otherwise indicated.

\title{
The association between leisure-time physical activity and lung function in older adults: The English longitudinal study of ageing
}

PLEASE CITE THE PUBLISHED VERSION

https://doi.org/10.1016/j.ypmed.2017.10.030

\section{PUBLISHER}

(C) Elsevier

VERSION

AM (Accepted Manuscript)

\section{PUBLISHER STATEMENT}

This work is made available according to the conditions of the Creative Commons Attribution-NonCommercialNoDerivatives 4.0 International (CC BY-NC-ND 4.0) licence. Full details of this licence are available at: https://creativecommons.org/licenses/by-nc-nd/4.0/

\section{LICENCE}

CC BY-NC-ND 4.0

\section{REPOSITORY RECORD}

O'Donovan, Gary, and Mark Hamer. 2019. "The Association Between Leisure-time Physical Activity and Lung Function in Older Adults: The English Longitudinal Study of Ageing". figshare. https://hdl.handle.net/2134/27318. 
The association between leisure-time physical activity and lung function in older adults: The English Longitudinal Study of Ageing

Gary O'Donovan, Ph.D. and Mark Hamer, Ph.D.

School of Sport, Exercise and Health Sciences, National Centre for Sport \& Exercise Medicine - East Midlands, Loughborough University, Loughborough LE11 3TU, United Kingdom.

Correspondence: Dr Gary O'Donovan, School of Sport, Exercise and Health Sciences, National Centre for Sport \& Exercise Medicine - East Midlands, Loughborough University, Loughborough LE11 3TU, United Kingdom. Email: g.odonovan@lboro.ac.uk

Word count, main text: 2,399

Word count, abstract: 250 


\begin{abstract}
The longitudinal association between physical activity and lung function is unclear.

Therefore, we examined said association over eight years. This study included data from 2,966 participants in English Longitudinal Study of Ageing (63 \pm 7 years [mean $\pm S D$ ]), a prospective study of initially healthy, community dwelling adults. Physical activity was assessed using an interview and lung function using a spirometer at baseline (2004-5) and follow-up (2012-13). General linear regression was used to assess associations between activity and lung function. Logistic regression was used to assess the odds of new cases of abnormal lung function. Some $14 \%$ of participants were defined as physically inactive at baseline, $50 \%$ were classified into the moderate group, and $36 \%$ into the vigorous group. In comparison with remaining inactive at follow-up, remaining active was positively associated with forced vital capacity (FVC) $(\beta=0.09,95 \%$ confidence interval $[\mathrm{Cl}]: 0.01,0.17 ; p=0.02)$ and forced expiratory volume in one second (FEV-1) $(\beta=0.09,95 \% \mathrm{Cl}: 0.02,0.15 ; p=0.01)$ after adjustment for baseline lung function score and other covariates. Using the fifth centile to define the lower limit of normal (that is, $-1.64 \mathrm{z}$ scores), there were lower odds of incident abnormal lung function in participants who remained physically active compared to those who remained inactive (FVC odds ratio $=0.31,95 \% \mathrm{Cl}$ : $0.17,0.55$. FEV-1 odds ratio=0.43, $95 \% \mathrm{Cl}: 0.26,0.72)$. Similar associations were observed in those who became active. This study suggests that remaining physically active or becoming active in older age are positively associated with lung function and reduced odds of abnormal lung function.
\end{abstract}




\section{Introduction}

The World Health Organization estimates that 65 million people have moderate to severe chronic obstructive pulmonary disease (COPD) and that more than three million people die per year because of the disease. ${ }^{1}$ Smoking is the main risk factor for COPD in high- and middle-income countries. ${ }^{2}$ Physical inactivity may also be a risk factor for COPD. ${ }^{3,4}$ Exercise training is recommended in the management of COPD. ${ }^{5}$ However, the role of physical activity in the primary prevention of COPD is unclear. ${ }^{6,7}$ In the 2014 European Respiratory Society statement on physical activity in COPD, ${ }^{6}$ five longitudinal studies were identified and each study showed an inverse association between physical activity and lung function decline in at least one population subgroup or physical activity variable. ${ }^{3,8-11}$ Nonetheless, the inverse association between physical activity and lung function decline was described as inconsistent. ${ }^{6}$ Selection bias, lack of adjustment for potential confounders, and lack of consideration of changes in physical activity in two of the five studies were identified as key limitations. ${ }^{6}$ There was no discussion of the role of physical activity in primary prevention in the 2017 Global Initiative for Chronic Obstructive Lung Disease. ${ }^{7}$ It is clear that more research is needed to understand the relationship between physical activity and lung function. In this study, we used a large population sample of community dwelling older adults to examine associations between changes in physical activity and lung function using contemporary spirometry prediction equations. 


\section{Methods}

\section{Participants}

The English Longitudinal Study of Ageing (ELSA) is an ongoing cohort study that contains a nationally representative sample of community dwelling men and women born on or before 29 February $1952 .{ }^{12}$ Data collected at wave two (2004-5) were used as the baseline for the present analysis as this was the first time clinical information was gathered. A clinical assessment was repeated eight years later (wave 6; 2012-13). Participants were excluded if they had eye or chest surgery during the three weeks prior to the assessment, or if they had been hospitalised for heart disease or stroke in the previous six weeks, or if they were pregnant, or if they had a tracheostomy. We also excluded participants reporting lung diseases. The London Multi-Centre Research Ethics Committee approved the study and participants gave written informed consent.

\section{Physical activity}

Self-reported physical activity was assessed at baseline. The interview included questions on the frequency of participation in moderate- and vigorous-intensity activities during leisure time (more than once per week; once per week; one to three times per month; hardly ever). As previously described, ${ }^{13}$ physical activity was then categorised into three groups: inactive (no moderate or vigorous activity); moderate activity at least once per week (but no vigorous); and vigorous activity at least once per week. The physical activity measure has demonstrated face validity in predicting various health outcomes. ${ }^{13,14}$ The same physical activity questions were asked six years later at wave 5 (2010-11) enabling us to model physical activity change. A binary physical activity variable (inactive or moderate versus vigorous activity) was created and change in physical activity over six years (waves 2 to 5) was categorized into four groups: remained inactive, became inactive, became active, or always active.

\section{Lung function}

Lung function was assessed using a spirometer at baseline (Escort, Vitalograph, Bucks, UK) and follow-up (NDD Easy On-PC, ndd Medical Technologies, Inc., Massachusetts, US). Forced expiratory volume in one second (FEV-1) and forced vital capacity (FVC) were assessed, as described in detail elsewhere. ${ }^{15}$ The nurse instructed the participant to perform a forced expiratory manoeuvre: the participant was told to stand up and remove any tight 
clothing; the participant was told, "You must try to blow out as much air as possible as hard and as fast as you can"; the nurse demonstrated the correct technique using a mouthpiece not connected to the spirometer and emphasised that the lips should be firmly wrapped around the mouthpiece; the nurse demonstrated a blow, pointing out afterwards the need for a full inspiration, a vigorous start to the exhalation, and sustained expiration; the participant was allowed at least one practice blow and was given feedback and encouragement as necessary. The protocol required three successful measurements to be completed and the highest satisfactory score was used. An unsatisfactory attempt was defined in the protocol. ${ }^{15}$ Briefly, an unsatisfactory blow included any of the following: an unsatisfactory start with excessive hesitation; laughing or coughing, especially during the first second; a Valsalva manoeuvre; leakage of air around the mouthpiece; obstruction of the mouthpiece by tongue or teeth; obstruction of the spirometer flowhead outlet by hands. Z-scores were calculated using the Global Lung Function 2012 Equations, ${ }^{16}$ which adjust for the heterogeneity of between-subject variability according to sex, ethnic group, age and lung function parameters. A z-score of zero would be assigned to a participant reaching their predicted lung function value, and the use of the 5 th centile has been recommended to define the lower limit of normal (that is, -1.64 z-scores).

\section{Covariates}

Nurses measured participants' body weight without shoes and in light clothing to the nearest $0.1 \mathrm{~kg}$ using electronic scales (THD-305 scales, Tanita Europe, Amsterdam, The Netherlands), and height was measured in the Frankfurt plane using a stadiometer. Body mass index (BMI) was calculated as weight (kilograms)/height (meters) squared. Handgrip strength $(\mathrm{kg})$ of the dominant hand was assessed using the Smedley hand-held dynamometer (Stoelting Co, IL, USA), using the average of three measurements. Participants were required to hold the device at a right angle to their body and exert maximum force for a couple of seconds when instructed. Health-related questions included cigarette smoking (current, previous or non-smoker), social occupational class (managerial and professional; intermediate; semi-routine and routine occupations), and clinician diagnosed cardiovascular diseases.

\section{Analysis}

Three sets of primary analyses were conducted to examine associations between physical activity and lung function, all using general linear regression. Firstly, the cross-sectional association between physical activity and lung function (z-score) at baseline was examined. 
The models were adjusted for age, sex, smoking (current, previous or non-smoker), social occupational class (managerial and professional; intermediate; semi-routine and routine occupations), BMI, grip strength, and self-reported physician diagnosis of cardiovascular disease at baseline. Secondly, the longitudinal association between physical activity at baseline lung function at follow-up was examined. Since we were interested in the variability between repeated measures of subjects, not in the variability between subjects we modelled the raw scores in these analyses (not Z-scores). The models were adjusted for the same set of covariates described above, with the addition of the respective lung function data at baseline to model change. Lastly, we examined the association of change in physical activity on lung function at follow-up using linear regression adjusting for the covariates described above. In separate analyses, logistic regression was used to investigate the odds of new cases of abnormal lung function. The fifth centile was used to define the lower limit of normal (that is, -1.64 z-scores), and we examined the association between change in physical activity and incident cases of abnormal lung function. Covariates were selected a priori based on evidence linking these covariates to both physical activity and ageing outcomes. ${ }^{13,14}$ One set of secondary analyses was conducted to examine effect modification by sex and smoking. All analyses were conducted using SPSS (IBM inc, version 22) with statistical significance $p<0.05$. 


\section{Results}

At baseline, 4,348 participants provided a lung function measure although 1,382 were lost to follow-up leaving a final analytic sample of 2,966 (1,358 men; $63 \pm 7$ years of age at baseline $[$ mean $\pm S D])$. Compared to the analytic sample, those who were excluded recorded poorer lung function (FEV-1 z-score: -0.75 vs. $-0.58, p<0.001$; FVC z-score: -0.53 vs. -0.32 , $\mathrm{p}<0.001$ ), were older (67.5 vs. $63.3 \mathrm{yrs}, \mathrm{p}<0.001)$, had higher BMI (28.2 vs. $27.9 \mathrm{~kg} . \mathrm{m}-2$, $p=0.023$ ), lower grip strength ( $28.7 \mathrm{vs} .31 .4 \mathrm{~kg}, p<0.001$ ), a higher prevalence of inactivity (22.1 vs. $13.0 \%, p<0.001$ ), and CVD (27.4 vs. $21.5 \%, p<0.001)$. The characteristics of the sample at baseline are presented in Table 1. Some 12\% of men and 15.5\% of women were categorised as physically inactive; some $49 \%$ of men and $51.5 \%$ of women were classified into the moderate physical activity group; and, some $39 \%$ of men and $33 \%$ of women were classified into the vigorous physical activity group.

There was a reduction in absolute FEV-1 $(2.52 \pm 0.82$ vs. $2.30 \pm 0.72 \mathrm{~L}, \mathrm{p}<0.001)$ and FVC values ( $3.41 \pm 1.04$ vs. $3.21 \pm 0.95 \mathrm{~L}, \mathrm{p}<0.001$ ) between baseline and follow-up, respectively. Table 2 shows the cross-sectional association between physical activity and lung function at baseline. Vigorous physical activity was positively associated with FEV-1 and FVC after adjustment for all covariates, whilst moderate physical activity was only associated with FVC. Table 3 shows null associations between baseline physical activity and lung function at 8 years of follow-up using the raw lung function data. In stepwise regression predicting FEV-1 at follow-up, baseline FEV-1, smoking, CVD, BMI, age, and sex were retained in the final model, predicting $42 \%$ of the variance (39.5\% predicted by baseline FEV-1). For analyses of FVC at follow-up, baseline FVC, smoking, CVD, BMI, age, sex, physical activity and grip strength were retained in the final model, predicting $35 \%$ of the variance $(31.2 \%$ predicted by baseline FVC). Table S1 and Table S2 in the online supplement show that there was no indication of effect modification by sex or smoking.

In the next set of analyses we examined associations between physical activity change over six years and lung function at follow-up (Table 4). The results show that participants who had become active or remained active had higher lung function scores at follow-up. Using the 5 th centile to define the lower limit of normal (that is, -1.64 z-scores), there were 200 and 132 new cases of abnormal lung function at follow-up based on FEV-1 and FVC scores, respectively (after removing participants with lung function scores below -1.64 z-scores at baseline). In logistic regression models there were lower odds of incident abnormal lung function in participants who remained physically active and those who became active (Table 5). 


\section{Discussion}

The objective of this longitudinal study was to test the notion that there is an inverse relationship between physical activity and lung function decline. The results suggest that regular participation in physical activity is positively associated with lung function at baseline. However, the association did not persist in longitudinal models. The main novelty of this work was to model changes in physical activity in relation to lung function. When we modelled change in physical activity, participants who had become active or remained active had higher lung function scores at follow-up after accounting for baseline lung function, and were less likely to have dropped into the lower limit of normal. These data support the notion that there is a beneficial relationship between becoming physically active and better lung function and the notion that there is a beneficial relationship between remaining physically active and better lung function. However, we cannot entirely rule out the possibility of reverse causation in that better lung function causes participants to be more active.

There was a beneficial association between at least one measure of physical activity and at least one measure of lung function in each of the five longitudinal studies cited in the pertinent European Respiratory Statement; ${ }^{6}$ however, the associations were not consistent and the need for more research was apparent, particularly in considering changes in physical activity level during follow-up. Nystad and colleagues ${ }^{17}$ have since reported that maintenance of physical activity over a 10-year period was inversely associated with lung function decline in 8047 men and women, although lung function was only measured once at follow-up making it impossible to examine true changes. Inconsistencies in the existing evidence may also be explained by differences in the methods used to analyse spirometry data. It is not clear what mechanisms might explain beneficial associations between physical activity and lung function or between physical activity and COPD. ${ }^{3}$ It has been suggested that physical activity has beneficial effects on respiratory muscle strength ${ }^{8,9}$ and that physical activity reduces the inflammation associated with COPD. ${ }^{3}$ Data from the ELSA cohort suggest that markers of inflammation explain around $15 \%$ of the association between sedentary behavior and mortality. ${ }^{18}$ Data from the Whitehall II cohort suggest that regular physical activity is associated with lower markers of inflammation over 10 years of followup. ${ }^{19}$ Smoking is the main risk factor for COPD ${ }^{2}$ and the United Kingdom government banned smoking in enclosed public places and in the workplace in 2007. Physical inactivity may also be a risk factor for chronic obstructive pulmonary disease; ${ }^{3,4}$ however, relatively little has been done to tackle physical inactivity. ${ }^{20-22}$ 
The main strengths of the present study are the relatively large number of participants, the assessment of physical activity change at follow-up, and the relatively long duration of follow-up. We removed participants with existing lung diseases at baseline to guard against reverse causation (ie, poor lung function causing low physical activity), although since these data are observational we cannot infer causality. The analyses were adjusted for a range of relevant covariates although we cannot discount the possibility of residual confounding. Analyses were adjusted for smoking status (current, previous or non-smoker), but not smoking volume. Smokers did report a median consumption of 24 cigarettes per week, but smoking volume did not influence any of the results (smoking volume defined according to the median split: non-smokers; light smokers, up to 24 cigarettes per week; heavy smokers, 24 or more cigarettes per week) (results not shown). It was not possible to investigate the influence of smoking cessation because quitting was only reported in 44 participants during follow-up. A further limitation of the study is the self-reported nature of physical activity. The dose-response relationship between physical activity intensity and lung function at baseline may be subject to recall bias, given that vigorous-intensity activities may be recalled with greater accuracy than moderate-intensity activities. ${ }^{23}$ Physical activity monitors and physical activity questionnaires have their advantages and disadvantages and questionnaires are still regarded as the mainstay of established longitudinal studies such as ELSA. ${ }^{24}$ The interviewer-led physical activity questionnaire in ELSA is relatively crude and cannot be used to investigate the benefits associated with adherence to prevailing physical activity guidelines. There was no statistically significant evidence of effect modification by sex and smoking; however, the null findings of these secondary analyses may be due to the relatively small sample sizes. More experimental evidence is required to clarify the effect of exercise intensity on lung function. 


\section{Conclusion}

This observational study adds to the existing evidence that maintenance and uptake of physical activity are beneficially associated with lung function in older adults. 


\section{Contributors' statement}

Hamer conceived the study and carried out the statistical analysis. O'Donovan and Hamer drafted the paper. Hamer had full access to the data, and takes responsibility for the integrity and accuracy of the results. Both authors contributed intellectually to refine the study design and contributed to the critical revision of the manuscript.

\section{Role of the funding source}

O'Donovan and Hamer acknowledge support from the National Institute for Health Research (NIHR) Leicester Biomedical Research Centre, which is a partnership between University Hospitals of Leicester NHS Trust, Loughborough University and the University of Leicester. The views expressed are those of the authors and not necessarily those of the NHS, the NIHR, the Department of Health. Hamer conceived the study and carried out the statistical analysis. O'Donovan and Hamer drafted the paper. The English Longitudinal Study of Ageing is funded by the UK Government departments, Department of Health, Department for Work and Pensions, and Department for Transport. Waves 7 and 8 were also supported by the US National Institute on Ageing. The present study received no specific funding.

\section{Conflicts of interest}

The authors have nothing to disclose. 
Table 1. Characteristics of the sample at baseline*

\begin{tabular}{lll}
\hline & Men $(\mathrm{n}=1358)$ & Women (n=1609) \\
\hline Age, years & $62.9 \pm 7.3$ & $63.3 \pm 7.4$ \\
Physical activity, \% & \\
$\quad$ Inactive & 12.3 & 15.5 \\
$\quad$ Moderate & 48.7 & 51.5 \\
$\quad$ Vigorous & 39.1 & 33.0 \\
Smoking, \% & 12.8 & 12.3 \\
Social occupation group & & \\
$\quad$ Managerial/ professional & 46.1 & 30.8 \\
$\quad$ Intermediate & 20.8 & 30.2 \\
$\quad$ Semi-routine /routine & 32.7 & 37.9 \\
Prevalent CVD, \% & 25.8 & 18.0 \\
FEV-1, L [z-score] & $3.0 \pm 0.8[-0.59 \pm 1.34]$ & $2.1 \pm 0.6[-0.58 \pm 1.31]$ \\
FVC, L [Z-score] & $4.1 \pm 1.0[-0.34 \pm 1.27]$ & $2.8 \pm 0.7[-0.29 \pm 1.39]$ \\
Body mass index, kg·m-2 & $27.9 \pm 4.1$ & $28.0 \pm 5.2$ \\
Grip strength, kg & $40.4 \pm 8.9$ & $23.8 \pm 6.2$ \\
\hline
\end{tabular}

*FEV1 is forced expiratory volume in one second; FVC is forced vital capacity. 
Table 2. Cross-sectional association between physical activity and lung function z-score at baseline $(n=2,966)^{\star}$

\begin{tabular}{lll}
\hline Physical activity group & FEV-1, $\beta(95 \% \mathrm{Cl})$ & FVC, $\beta(95 \% \mathrm{Cl})$ \\
\hline Inactive $(\mathrm{n}=384)$ & Reference & Reference \\
Moderate $(\mathrm{n}=1,481)$ & $0.12(-0.02,0.27)$ & $0.21(0.06,0.36)$ \\
Vigorous $(\mathrm{n}=1,101)$ & $0.26(0.11,0.42)$ & $0.32(0.16,0.47)$ \\
$\mathrm{P}$, trend & 0.001 & 0.001 \\
\hline
\end{tabular}

*Values are unstandardised beta coefficient $(\beta)$ and 95\% confidence interval $(\mathrm{Cl})$, adjusted for age, sex, smoking habit (current, previous or non-smoker), social occupational class, body mass index, grip strength of the dominant hand, and self-reported physician diagnosis of cardiovascular disease. FEV1 is forced expiratory volume in one second; FVC is forced vital capacity. FEV1, FVC were treated as z-scores using the Global Lung Function 2012 Equations. 
Table 3. Longitudinal association between physical activity at baseline and lung function at follow-up $(n=2,966)^{*}$

\begin{tabular}{lll}
\hline Physical activity group & FEV-1, $\beta(95 \% \mathrm{Cl})$ & FVC, $\beta(95 \% \mathrm{Cl})$ \\
\hline Inactive $(\mathrm{n}=384)$ & Reference & Reference \\
Moderate $(\mathrm{n}=1,481)$ & $0.02(-0.03,0.06)$ & $-0.02(-0.07,0.04)$ \\
Vigorous $(\mathrm{n}=1,101)$ & $0.04(-0.004,0.09)$ & $0.05(-0.01,0.11)$ \\
$\mathrm{P}$, trend & 0.043 & 0.012 \\
\hline
\end{tabular}

*Values are unstandardised beta coefficients $(\beta)$ and 95\% confidence interval $(\mathrm{Cl})$, adjusted for age at baseline, sex, the respective lung function reading at baseline, smoking habit at baseline (current, previous or non-smoker), Social occupational class, body mass index at baseline, grip strength of the dominant hand at baseline, and self-reported physician diagnosis of cardiovascular disease at baseline. FEV1 is forced expiratory volume in one second; FVC is forced vital capacity. 
Table 4. Association between change in physical activity and lung function at follow-up $(n=2,874)^{*}$

\begin{tabular}{lll}
\hline Physical activity group & FEV-1, $\beta(95 \% \mathrm{Cl})$ & FVC, $\beta(95 \% \mathrm{Cl})$ \\
\hline Remain inactive $(\mathrm{n}=152)$ & Reference & Reference \\
Became inactive $(\mathrm{n}=364)$ & $0.05(-0.02,0.11)$ & $0.02(-0.07,0.11)$ \\
Became active $(\mathrm{n}=215)$ & $0.09(0.01,0.16)$ & $0.12(0.02,0.21)$ \\
Remain active $(\mathrm{n}=2143)$ & $0.09(0.02,0.15)$ & $0.09(0.01,0.17)$ \\
\hline
\end{tabular}

*Values are unstandardised beta coefficients $(\beta)$ and 95\% confidence interval $(\mathrm{Cl})$, adjusted for age at baseline, sex, the respective lung function reading at baseline, smoking habit at baseline (current, previous or non-smoker), Social occupational class, body mass index at baseline, grip strength of the dominant hand at baseline, and self-reported physician diagnosis of cardiovascular disease at baseline. FEV1 is forced expiratory volume in one second; FVC is forced vital capacity. 
Table 5. Associations between change in physical activity and incident abnormal lung function (participants with z-score less than -1.64 at baseline removed)*

\begin{tabular}{lll}
\hline Physical activity group & $\begin{array}{l}\text { Abnormal FEV-1, } \\
\text { OR }(95 \% \mathrm{Cl})\end{array}$ & $\begin{array}{l}\text { Abnormal FVC, } \\
\text { OR }(95 \% \mathrm{Cl})\end{array}$ \\
\hline Remain inactive & 1.0 (reference) & 1.0 (reference) \\
Became inactive & $0.56(0.31,1.03)$ & $0.34(0.17,0.70)$ \\
Became active & $0.25(0.10,0.58)$ & $0.35(0.16,0.80)$ \\
Remain active & $0.43(0.26,0.72)$ & $0.31(0.17,0.55)$ \\
\hline
\end{tabular}

*Values are are odds ratios (OR) and 95\% confidence interval $(\mathrm{Cl})$, adjusted for age at baseline, sex, smoking habit at baseline (current, previous or non-smoker), social occupational class, body mass index at baseline, grip strength of the dominant hand at baseline, and self-reported physician diagnosis of cardiovascular disease at baseline. FEV1 is forced expiratory volume in one second; FVC is forced vital capacity. The 5th centile was used to define the lower limit of normal lung function (i.e. -1.64 z-scores). 


\section{References}

1. World Health Organization. Burden of COPD. 2017.

http://www.who.int/respiratory/copd/burden/en/ (accessed August 2017).

2. World Health Organization. Causes of COPD. 2017.

http://www.who.int/respiratory/copd/causes/en/ (accessed August 2017).

3. Garcia-Aymerich J, Lange P, Benet M, Schnohr P, Anto JM. Regular physical activity modifies smoking-related lung function decline and reduces risk of chronic obstructive pulmonary disease: a population-based cohort study. Am J Respir Crit Care Med 2007; 175(5): 458-63.

4. Behrens G, Matthews CE, Moore SC, Hollenbeck AR, Leitzmann MF. Body size and physical activity in relation to incidence of chronic obstructive pulmonary disease. CMAJ 2014; 186(12): E457-69.

5. World Health Organization. COPD management. 2017. http://www.who.int/respiratory/copd/management/en/ (accessed August 2017).

6. Watz H, Pitta F, Rochester CL, et al. An official European Respiratory Society statement on physical activity in COPD. Eur Respir J 2014; 44(6): 1521-37.

7. Global Initiative for Chronic Obstructive Lung Disease (GOLD). Global Strategy for the Diagnosis, Management and Prevention of COPD. 2017. http://goldcopd.org/ (accessed February 2017).

8. Pelkonen M, Notkola IL, Lakka T, Tukiainen HO, Kivinen P, Nissinen A. Delaying decline in pulmonary function with physical activity: a 25-year follow-up. Am J Respir Crit Care Med 2003; 168(4): 494-9.

9. Jakes RW, Day NE, Patel B, et al. Physical inactivity is associated with lower forced expiratory volume in 1 second : European Prospective Investigation into Cancer-Norfolk Prospective Population Study. Am J Epidemiol 2002; 156(2): 139-47. 
10. Cheng YJ, Macera CA, Addy CL, Sy FS, Wieland D, Blair SN. Effects of physical activity on exercise tests and respiratory function. Br J Sports Med 2003; 37(6): 521-8.

11. Garcia-Aymerich J, Lange P, Serra I, Schnohr P, Anto JM. Time-dependent confounding in the study of the effects of regular physical activity in chronic obstructive pulmonary disease: an application of the marginal structural model. Ann Epidemiol 2008; 18(10): 775-83.

12. Steptoe A, Breeze E, Banks J, Nazroo J. Cohort profile: the English longitudinal study of ageing. Int J Epidemiol 2013; 42(6): 1640-8.

13. Hamer M, Molloy GJ, de Oliveira C, Demakakos P. Leisure time physical activity, risk of depressive symptoms, and inflammatory mediators: the English Longitudinal Study of Ageing. Psychoneuroendocrinology 2009; 34(7): 1050-5.

14. Hamer M, de Oliveira C, Demakakos P. Non-exercise physical activity and survival: English longitudinal study of ageing. Am J Prev Med 2014; 47(4): 452-60.

15. ELSA. IP2158/P8158 ELSA Wave Two Nurse Visit. Project Instructions. 2004. http://www.elsa-project.ac.uk/documentation (accessed December 2016).

16. Quanjer PH, Stanojevic S, Cole TJ, et al. Multi-ethnic reference values for spirometry for the 3-95-yr age range: the global lung function 2012 equations. Eur Respir J 2012; 40(6): 1324-43.

17. Nystad W, Samuelsen SO, Nafstad P, Langhammer A. Association between level of physical activity and lung function among Norwegian men and women: the HUNT study. Int J Tuberc Lung Dis 2006; 10(12): 1399-405.

18. Hamer M, Yates T, Demakakos P. Television viewing and risk of mortality: Exploring the biological plausibility. Atherosclerosis 2017; 263: 151-5.

19. Hamer M, Sabia S, Batty GD, et al. Physical Activity and Inflammatory Markers Over 10 Years: Follow-Up in Men and Women From the Whitehall II Cohort Study. Circulation 2012; 126(8): 928-33. 
20. Fox KR, Hillsdon M. Physical activity and obesity. Obes Rev 2007; 8 Suppl 1: 11521.

21. Wareham N. Physical activity and obesity prevention. Obes Rev 2007; 8 Suppl 1: 109-14.

22. Reis RS, Salvo D, Ogilvie D, et al. Scaling up physical activity interventions worldwide: stepping up to larger and smarter approaches to get people moving. Lancet 2016; 388(10051): 1337-48.

23. Lee IM, Paffenbarger R, S, Jr. How Much Physical Activiry is Optimal for Health? Methodological Considerations. Res Q Exerc Sport 1996; 67(2): 206-8.

24. Pedisic Z, Bauman A. Accelerometer-based measures in physical activity surveillance: current practices and issues. Br J Sports Med 2015; 49(4): 219-23. 